\title{
A Chandra Study: Are Dwarf Carbon Stars Spun Up and Rejuvenated by Mass Transfer?
}

\author{
Paul J. Green ${ }^{1}\left(\mathbb{D}\right.$, Rodolfo Montez ${ }^{1}$ (D), Fernando Mazzoni ${ }^{2}$, Joseph Filippazzo ${ }^{3}$ (i), Scott F. Anderson ${ }^{4}$, Orsola De Marco ${ }^{5,6}$, \\ Jeremy J. Drake ${ }^{1}$ (1) , Jay Farihi ${ }^{7}$ (10), Adam Frank ${ }^{8}$, Joel H. Kastner, ${ }^{9,10}$ (1) Brent Miszalski ${ }^{1,12}$, and Benjamin R. Roulston ${ }^{1,13}$ (1) \\ ${ }^{1}$ Harvard Smithsonian Center for Astrophysics, 60 Garden Street, Cambridge, MA 02138, USA; pgreen@cfa.harvard.edu \\ ${ }^{2}$ University of Massachusetts, Lowell, MA, USA \\ ${ }^{3}$ Space Telescope Science Institute, 3700 San Martin Drive, Baltimore, MD 21218, USA \\ ${ }^{4}$ Department of Astronomy, University of Washington, Box 351580, Seattle, WA 98195, USA \\ ${ }_{5}^{5}$ Department of Physics \& Astronomy, Macquarie University, Sydney, NSW 2109, Australia \\ ${ }^{6}$ Astronomy, Astrophysics and Astrophotonics Research Centre, Macquarie University, Sydney, NSW 2109, Australia \\ ${ }_{7}^{7}$ Department of Physics and Astronomy, University College London, London WC1E 6BT, UK \\ ${ }^{8}$ Department of Physics and Astronomy, University of Rochester, Rochester, NY 14627-0171, USA \\ ${ }^{9}$ Chester F. Carlson Center for Imaging Science, Rochester Institute of Technology, 54 Lomb Memorial Drive, Rochester, NY 14623, USA \\ ${ }^{10}$ School of Physics \& Astronomy and Laboratory for Multiwavelength Astrophysics, Rochester Institute of Technology, 54 Lomb Memorial Drive, Rochester, NY \\ 14623, USA \\ ${ }^{11}$ South African Astronomical Observatory, P.O. Box 9, Observatory 7935, South Africa \\ ${ }^{12}$ Southern African Large Telescope Foundation, P.O. Box 9, Observatory 7935, South Africa \\ ${ }^{13}$ Department of Astronomy, Boston University, 725 Commonwealth Avenue, Boston, MA 02215, USA \\ Received 2019 May 13; revised 2019 June 20; accepted 2019 June 21; published 2019 August 12
}

\begin{abstract}
Carbon stars (with $\mathrm{C} / \mathrm{O}>1$ ) were long assumed to all be giants, because only asymptotic giant branch (AGB) stars dredge up significant carbon into their atmospheres. The case is nearly ironclad now that the formerly mysterious dwarf carbon $(\mathrm{dC})$ stars are actually far more common than $\mathrm{C}$ giants and have accreted carbon-rich material from a former AGB companion, yielding a white dwarf (WD) and a dC star that has gained both significant mass and angular momentum. Some such $\mathrm{dC}$ systems have undergone a planetary nebula phase, and some may evolve to become $\mathrm{CH}$, CEMP, or Ba giants. Recent studies indicate that most dCs are likely from older, metal-poor kinematic populations. Given the well-known anticorrelation of age and activity, dCs would not be expected to show significant X-ray emission related to coronal activity. However, accretion spin-up might be expected to rejuvenate magnetic dynamos in these post-mass-transfer binary systems. We describe our Chandra pilot study of six dCs selected from the SDSS for $\mathrm{H} \alpha$ emission and/or a hot WD companion, to test whether their $\mathrm{X}$-ray emission strength and spectral properties are consistent with a rejuvenated dynamo. We detect all six dCs in the sample, which have X-ray luminosities ranging from $\log L_{\mathrm{x}} \sim 28.5-29.7$, preliminary evidence that dCs may be active at a level consistent with stars that have short rotation periods of several days or less. More definitive results require a sample of typical dCs with deeper X-ray observations to better constrain their plasma temperatures.
\end{abstract}

Key words: binaries: general - stars: carbon - stars: chemically peculiar - X-rays: stars

\section{Introduction}

\subsection{Dwarf Carbon Stars}

Carbon (C) stars show molecular absorption bands of carbon- $\mathrm{C}_{2}, \mathrm{CN}$, or $\mathrm{CH}$ in their optical spectra-because they have $\mathrm{C} / \mathrm{O}>1$, with atmospheres cool enough to form molecules. By contrast, the $\mathrm{C} / \mathrm{O}$ ratio for nearby Sun-like stars is between $\sim 0.5$ and 0.8 (Fortney 2012 and references therein). In most single stars of intermediate mass $\left(\sim 1-8 M_{\odot}\right)$, atmospheres show $\mathrm{C} / \mathrm{O}$ above unity only in the asymptotic giant branch (AGB) phase, when carbon is brought to the surface during episodes of strong convection. The so-called third dredge-up occurs during late stages of thermal pulsations (the TP-AGB phase), associated with the triple- $\alpha$ process in shell He-burning episodes. AGB stars may reach a radius of $\sim 1$ au $\left(\sim 200 R_{\odot}\right)$. Pulsations, shocks, and radiation all drive carbon- and $s$-process-enhanced material outward in an AGB wind, which may result in detached shells of circumstellar material containing $50 \%-70 \%$ of the star's mass (Wood et al. 2004). Winds from AGB stars are the predominant source of carbon in the interstellar medium, the PAHs in star-forming regions, prebiotic molecules, and indeed the planets as well (e.g., Tielens 2005).

The discovery of the first main-sequence carbon star G77-61 $\left(M_{V}=10.1\right.$; Dahn et al. 1977) sounded like an oxymoron at the time. Dahn et al. (1977) offered several possible explanations for the existence of a dwarf carbon (dC) star. That G77-61 is in a binary system $(P \sim 245$ days; Dearborn et al. 1986) with an unseen companion consistent with a cool white dwarf (WD) strongly suggested that extrinsic processes produce the dC's enhanced atmospheric abundances. Recent results from radial velocity monitoring of larger samples of dCs are consistent with a 100\% binary fraction (Whitehouse et al. 2018; Roulston et al. 2019). Therefore, the favored hypothesis to explain $\mathrm{dCs}$ is that C-rich material lost in an AGB wind can be efficiently captured by a main-sequence companion. While the AGB donor has since evolved to a WD, the "polluted" companion, as an innocent bystander, could be either dwarf or giant in the current epoch. Indeed, a variety of non-AGB stars, including many red giant stars, show enhanced carbon and/or $s$-process abundances that are similarly extrinsic. The $\mathrm{CH}, \mathrm{Ba}$, and the carbon-enhanced metal-poor (CEMP-s) stars (Lucatello et al. 2005)—mostly 
giants or subgiants-likely evolved from dC stars, and have been more commonly studied only by virtue of their greater brightness. All these objects can show atmospheric signatures of $s$-process elements associated with neutron capture onto iron seed nuclei in AGB stars.

Is there another viable path to strong carbon enhancement besides mass transfer? Intriguingly, the CEMP-no class lacks $s$ process overabundances, and initial radial velocity studies do not show an enhanced binary fraction (Starkenburg et al. 2014), so CEMP-no stars may achieve atmospheric carbon enhancement without mass transfer. Debate continues, but one suggestion is that (reminiscent of one of the suggestions in Dahn et al. 1977) CEMP-no stars may have coalesced from clouds seeded with strong carbon overabundances (and insignificant iron) by the first zero-heavy-element stars (e.g., Bromm \& Loeb 2003; Norris et al. 2013). This interpretation is favored by the fact that nearly all CEMP-no stars have $[\mathrm{Fe} / \mathrm{H}] \leqslant-4.0$. The prototype $\mathrm{dC}$ star, G77-61, has a companion, but also has extremely low metallicity $([\mathrm{Fe} / \mathrm{H}] \sim-4$; Plez \& Cohen 2005), so might be a candidate for either mechanism.

The importance of dC stars like G77-61 was later confirmed as many additional dCs were recognized from their large proper motions (Green et al. 1991; Downes et al. 2004). In a handful of cases, the "smoking gun" of AGB binary mass transfer was revealed as a hot DA WD companion (Heber et al. 1993; Liebert et al. 1994). More recently Green (2013) found more than $700 \mathrm{dC}$ stars in the SDSS, powerfully confirming that $\mathrm{dCs}$ are in fact the numerically dominant type of carbon star in the Galaxy, supporting the ubiquity of the $\mathrm{dC}$ phenomenon across a broad range of mass and age. Other large samples have since emerged from LAMOST (Ji et al. 2016; Li et al. 2018), including a dozen double-line spectroscopic binary (SB2) DA $+\mathrm{dC}$ spectra, which reveal recently minted systems ( $\mathrm{Si}$ et al. 2014). Dwarf carbon stars show kinematic evidence of mostly belonging to older populations (Green 2013; Farihi et al. 2018). Dwarf carbon stars may be favored in older e.g., thick disk or halo, systems because they are born with large $\mathrm{C} / \mathrm{O}$ (as for the CEMP-no hypothesis above) or simply because at lower metallicity, less mass transfer is required to boost $\mathrm{C} / \mathrm{O}>1$.

Dozens of dCs show Balmer emission lines, signs of strong chromospheric activity. This is surprising, since activity is rare in older stars. Activity is also associated with enhanced X-ray emission, which for $\mathrm{dC}$ stars remains uncharacterized. Stellar activity - which correlates with rapid rotation in the general population (see Section 1.4)—may be a result of spin-up by accretion in $\mathrm{dC}$ stars, a hypothesis we aim to test here via X-ray observations.

\subsection{The Planetary Nebula Connection}

Once the hot core of an AGB star is revealed, it may illuminate the layers of expelled material to form a spectacular planetary nebula (PN). Binary companions to the central stars of $\mathrm{PNe}(\mathrm{CSPNe})$ are widely believed to be responsible for bipolar (axisymmetric) structures in PNe (see, e.g., Balick \& Frank 2002; Balick 2004; García-Segura et al. 2018). Many dC stars were likely born within planetary nebulae. Indeed, the CSPN of PN G054.2-03.4 ("The Necklace;" Miszalski et al. 2013) reveals a dC spectrum when the CSPN is in eclipse, offering direct evidence for this link between PNe and dC stars.

Surviving main-sequence companions to CSPNe may be "born-again"-resembling pre-main-sequence stars, but with rejuvenated coronae and (hence) luminous X-ray emission
(Soker \& Kastner 2002). Chandra imaging of CSPNe (Montez et al. 2010) has revealed that most of the pointlike X-ray sources are too hard to be modeled as blackbody emission from a pre-WD stellar photosphere ( $\sim 100-200 \mathrm{kK})$. Among CSPNe with hard X-ray emission $\left(T_{\mathrm{X}} \gtrsim>6 \mathrm{MK}\right.$ ), at least $75 \%$ (six out of eight) have known close or rapidly rotating companions (Kastner et al. 2012; Montez et al. 2015). The companions are expected to remain active much longer ( $>$ Gyr; West et al. 2008) than characteristic PN lifetimes $\left(\sim 10^{5} \mathrm{yr}\right.$; e.g., Frew 2008). While the nearest known binary CSPNe with hard $\mathrm{X}$-rays are at $\sim 500 \mathrm{pc}$ (NGC 1514 at $460 \pm 8 \mathrm{pc}$ and LoTr 5 at $499 \pm 12$ pc; Bailer-Jones et al. 2018), field dCs are worthy of study because some are as close as $\sim 80 \mathrm{pc}$ and uncontaminated in the X-rays by hot CSPN radiation.

\subsection{Binary Evolution Scenarios}

Mass transferred from the former AGB companion may occur via stellar wind, Roche-lobe overflow (RLOF), and/or commonenvelope (CE) evolution. Models for $\mathrm{dC}$ formation in both the disk and halo (de Kool \& Green 1995) predict a bimodal orbital period distribution, with a large peak near a decade, for objects that have accreted AGB wind material with no substantial decrease of the orbital separation. A smaller peak near 1 yr likely contains systems that underwent a $\mathrm{CE}$ phase, where the companion was subsumed in the expanding atmosphere of the AGB star when it filled its Roche lobe. These models reproduce the well-studied distributions of $\mathrm{CH}$ and $\mathrm{BaII}$ giants, whose progenitors are almost certainly the $\mathrm{dC}$ stars. However, many gaps remain in our understanding. For instance, using a variety of reasonable typical assumptions about the initial period distribution and the physics of the mass-transfer process, the synthetic populations of Abate et al. (2018) underpredict the frequency of short-period systems relative to an unbiased sample of observed CEMP-s stars (e.g., Iaconi et al. 2018).

Very small initial orbital separations may not readily result in $\mathrm{dC}$ stars, because the primary will fill its Roche lobe, truncating evolution before the TP-AGB phase. However, orbits can evolve in a variety of ways as described, e.g., by Chen et al. (2018). Recent results from radial velocity monitoring of $\mathrm{dCs}$ (Whitehouse et al. 2018; Roulston et al. 2019) find some dCs with unexpectedly short periods (1.2 days, Corradi et al. 2011; Miszalski et al. 2013; $\sim 3$ days, Margon et al. 2018) or show large RV variations with as-of-yet undetermined orbits (Roulston et al. 2019). The dCs may prove quite important for further studies, because we know directly from their $\mathrm{C}_{2}$ and $\mathrm{CN}$ bands that they are post-AGB mass-transfer binaries, which makes them especially valuable laboratories of stellar binary evolution.

\subsection{Stellar Rotation and Activity}

Since $\mathrm{dC}$ stars seem to be from older (thick disk or halo) populations (Green 2013; Farihi et al. 2018), their congenital rotation rates, dynamo strengths, and related activity should have wound down significantly (e.g., Gondoin 2018 and references therein). However, Jeffries \& Stevens (1996) describe how the accretion of a massive, slow $\left(10-20 \mathrm{~km} \mathrm{~s}^{-1}\right)$ AGB wind is expected to spin up its low-mass secondary to short $(\lesssim 10 \mathrm{hr})$ rotation periods, for final orbital separations of about $100 \mathrm{au}$ or less. We may therefore expect significant activity and X-ray emission to be characteristic of $\mathrm{dC}$ stars - at least those in similar orbits-even if their progenitors may be older than most active stars in the Galaxy. 
The activity lifetimes inferred for $\mathrm{M}$ dwarfs range from $\sim 1$ to 5 Gyr (for M1-M4 stars; West et al. 2008). The dC stars could remain active for similar timespans after mass transfer. The active fraction among late-type stars as a function of age, metallicity, and binary orbital elements is a current topic of strong interest (e.g., West et al. 2008; Morgan et al. 2012), but the strength, frequency, or duration of activity in rejuvenated (post-mass-transfer) dwarfs remains unknown. The dC stars offer a unique tracer, because even without measured orbital signatures of binarity, their $\mathrm{C}_{2}$ and $\mathrm{CN}$ bands mark them clearly as post-mass-transfer systems.

Depending on the host system metallicity and evolution, a dC star may have inherited a substantial fraction of its final mass by accretion (e.g., Miszalski et al. 2013), potentially changing its overall structure in the process. Assuming the accreting star is fully convective, then it must inherit $0.1-0.2 M_{\odot}$ of $\mathrm{C} / \mathrm{O} \sim 2-3$ material. By contrast a much smaller quantity of more highly enriched (e.g., $\mathrm{C} / \mathrm{O}>20$ ) material would suffice. As mentioned above, the metallicity of the accretor is also key; for a star like G77-61 with extremely low metallicity (Plez \& Cohen 2005), much less mass transfer is required from a donor with the same $\mathrm{C} / \mathrm{O}$.

After a major mass accretion event, the dwarf's radius may remain inflated by accretion shocks, or even by enhanced magnetic activity itself, as debated for CSPNe (e.g., Jones et al. 2015). Nevertheless, long after the newly minted dC settles back to the main sequence from a thermally unstable phase (with a radius potentially $\sim 2.5 \times$ greater than a normal $\mathrm{dC}$; Afşar \& Ibanoğlu 2008), we expect a long-lasting ( Gyr) active phase as the compact core cools and fades.

Rapid rotation rates are typically found in young stars, as long as they retain angular momentum from their initial collapse. Rapid rotation together with convection is thought to drive an internal magnetic dynamo and consequent magnetic activity (e.g., Kosovichev et al. 2013 and references therein). The tangling, breaking, and reconnection of magnetic field lines, as seen in the Sun, in turn generates chromospheric activity (associated observationally, e.g., with $\mathrm{H} \alpha$ emission) and coronal activity (associated with X-ray emission). These tracers of magnetic activity decrease with stellar rotation rates (e.g., Pallavicini et al. 1981; Wright et al. 2011; Reiners et al. 2012). Rotation periods of main-sequence stars with outer convection zones increase with age due to angular momentum loss through magnetized winds (e.g., Kraft 1967; Matt et al. 2015; Garraffo et al. 2018).

Even though we do not yet have rotation periods measured for $\mathrm{dCs}$, the detection of enhanced X-ray emission would provide very suggestive evidence that they have active dynamos, consistent with rotation rates enhanced by spin-up from the accretion of angular momentum during past episodes of post-AGB binary mass transfer. This possibility motivated the pilot Chandra X-ray study whose results we describe below.

\section{Sample Selection}

The list of six targets for this initial Chandra study of X-rays from $\mathrm{dC}$ stars was compiled from our uniformly selected SDSS sample of high-latitude C stars (Green 2013). We selected only those that are definitively main sequence, based on high proper motions measured between USNO-B and SDSS (Munn et al. 2004). (Any giant with such high proper motion would be nearby and much too bright for SDSS.) For the most resource-efficient exploration of $\mathrm{dC}$ stars, we restricted the sample to either $\mathrm{DA}+\mathrm{dC}$ systems or $\mathrm{dCs}$ showing $\mathrm{H} \alpha$ emission (dCe stars, henceforth) with $i<17$, yielding six objects (two $\mathrm{DA}+\mathrm{dCs}$, and four $\mathrm{dCes}$ ). One was already observed in X-rays; SDSS J125017.90+252427.6 falls serendipitously in both XMM-Newton and Chandra fields (Green 2013), as it is only about $6^{\prime}$ away from the galaxy NGC 4725.

The small pilot sample we consider here likely includes $\mathrm{dC}$ stars (1) with a relatively short time since accretion (the $\mathrm{dC}$ systems including still-hot DA WDs) and/or (2) those still showing clear signs of activity (the dCe stars, for which the presumed DA WD has cooled beyond detectability even in the optical/UV). SDSS spectra are shown in Figure 1 for the objects in our Chandra sample, illustrating the range of $\mathrm{dC}$ types herein.

For context, we make use of the distance information derived from Gaia Data Release 2 (Gaia Collaboration et al. 2018) by Bailer-Jones et al. (2018) to plot the range of color and absolute magnitude for a compilation of non-AGB carbon-enhanced stars in Figure 2. While originally selected based on their large proper motions, the $\mathrm{dC}$ systems in our sample are confirmed as definitively main sequence, having $M_{G}>8$.

\section{X-Ray Observations and Analysis}

Our targeted Chandra observations of $\mathrm{dC}$ stars were performed using the S3 (backside illuminated CCD) on ACIS-S between 2014 October and 2016 May (proposals 15200243 and 16200105; PI: P. Green). Exposure times ranged from 16 to $28 \mathrm{ks}$ per star. Some of the observations were split into several exposures (ObsIDs) as part of the requirement to keep various Chandra subsystem temperatures within their acceptable ranges. None of the observations uses a grating. Most are in VFAINT ACIS mode, but several are in FAINT mode; observation details are listed in Table 1.

For SDSS J163718.64+274026.5, we analyzed the merged observations, since they use the same instrument, and are only separated by two days (and just $4^{\circ}$ in roll angle). SDSS J125017.90+252427.6 falls within three archival observations, with significantly different dates and instrument configurations, so we chose to analyze the longest observation only (29.6 ks; PI: Garmire).

We reprocessed the Chandra event lists with the CIAO (ver 4.10) chandra_repro script and CALDB (4.7.8), which account for afterglows, bad pixels, charge transfer inefficiency, and time-dependent gain corrections. All our dCs were detected in every Chandra ObsID, at positions within $2^{\prime \prime}$ of those measured in Gaia DR2 (epoch 2015.5). We used the CIAO srcflux tool to estimate source properties in the $0.3-3.0 \mathrm{keV}$ energy range, which includes all detected photons. X-ray source properties are shown in Table 2. To estimate intervening absorption from extinction due to Milky Way dust, we used the dustmaps Python package (Bayestar17) of Green (2018); for every $\mathrm{dC}$ direction, these columns were inconsequential.

For each $\mathrm{dC}$, we derived two X-ray flux estimates, based on either a $2 \mathrm{MK}$ or a $10 \mathrm{MK}$ optically thin plasma (APEC; Smith et al. 2001) with absorption modeled using WABS (Morrison \& McCammon 1983). As described in section Appendix, our motivation for these choices comes both from the literature and from attempts to fit the Chandra spectra of the two dCs herein with the most counts. Further discussion of our sample below includes results assuming either 2 or $10 \mathrm{MK}$ plasma temperatures. 


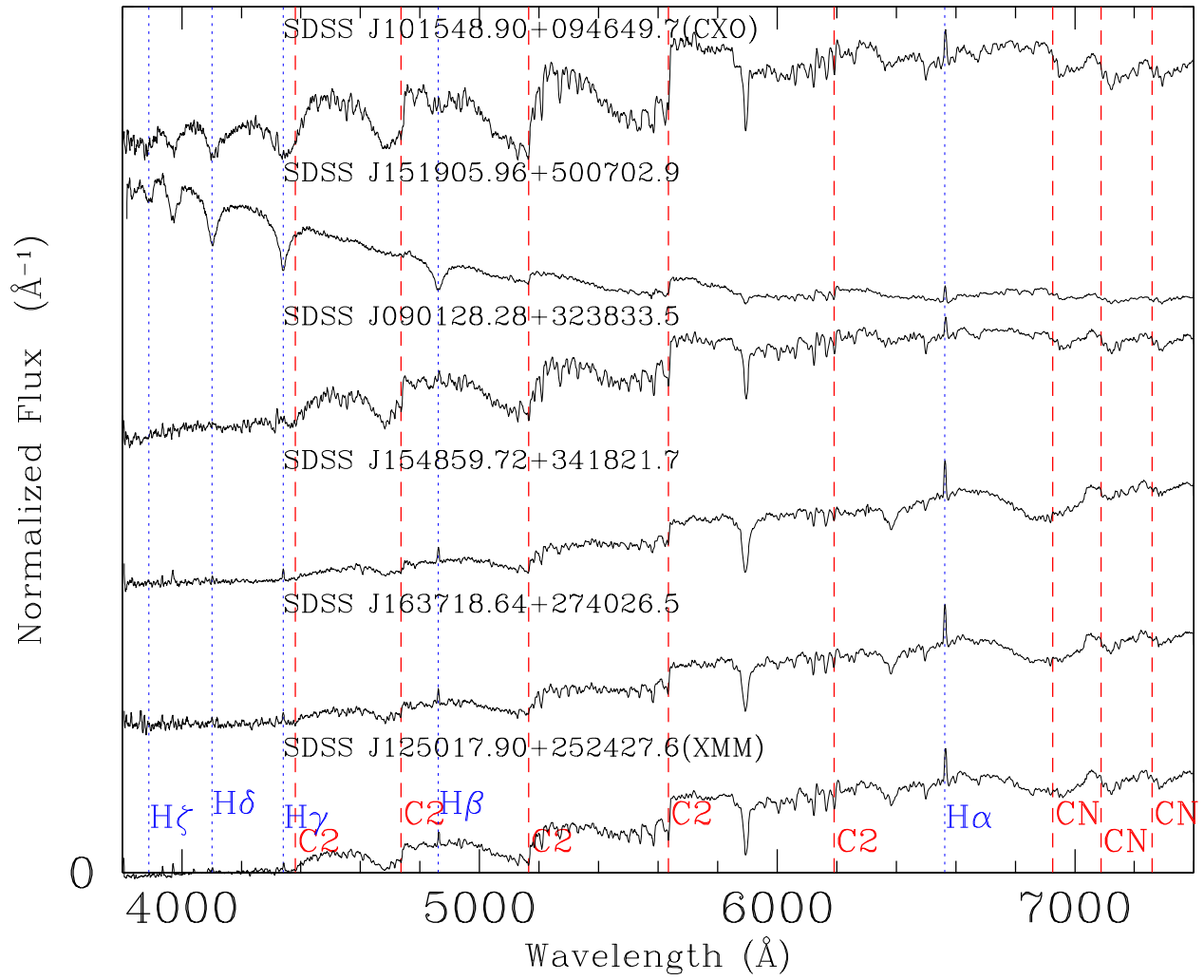

Figure 1. SDSS spectra of our full $\mathrm{dC}$ star sample. All show strong $\mathrm{C}_{2}$ bands at $\lambda 4737,5165$, and 5636 , as well as $\mathrm{H} \alpha$ emission betraying chromospheric activity. Broad Balmer absorption lines in the top two spectra reveal their hot white dwarf companions. $\mathrm{C}_{2}$ bandhead positions are shown with red long-dashed lines, and Balmer line wavelengths with blue short-dashed lines.

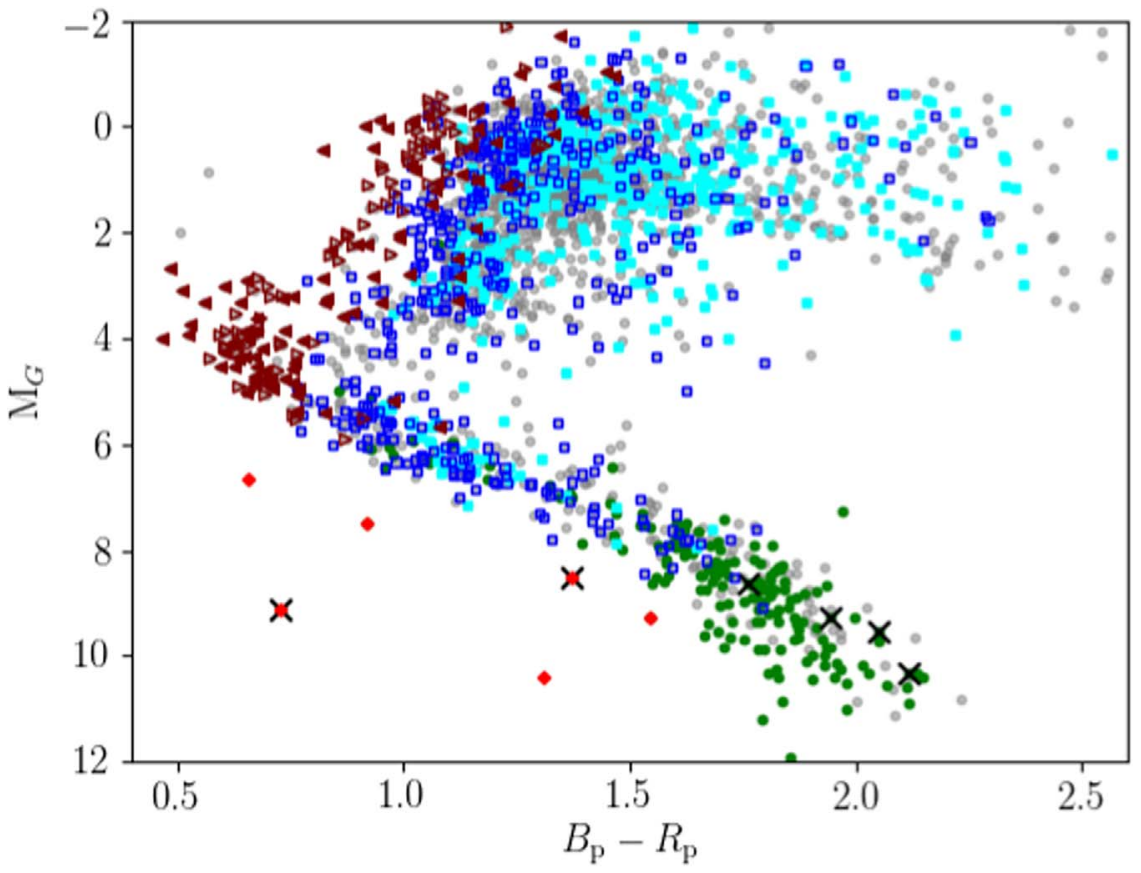

Figure 2. Color-magnitude diagram derived from our compilation of the latest spectroscopic samples of carbon-enhanced stars, using Gaia DR2 to derive an absolute $G$-band magnitude, and showing only significant parallaxes, with $\varpi / \sigma_{\varpi}>5$. Dwarf carbon (dC) stars, largely from SDSS (Green 2013; Si et al. 2014) and LAMOST (Yoon et al. 2016; Li et al. 2018), form the main sequence, $\left(6 \lesssim M_{G} \lesssim 11\right.$ ), most showing strong $\mathrm{C}_{2}$ and $\mathrm{CN}$ molecular bands. Dwarfs identified in Green (2013) are shown by filled green circles. Red diamonds mark known $\mathrm{DA} / \mathrm{dC}$ systems. The $\mathrm{dCs}$ observed with Chandra and analyzed herein are marked with large black crosses. $\mathrm{CH}$ (empty blue squares) and Ba II (filled cyan squares) from Li et al. (2018) and CEMP stars (maroon triangles) from Yoon et al. (2016) are also shown. As innocent bystanders to mass transfer, $\mathrm{dCs}$ are seen across a range of absolute magnitudes and temperatures, as long as they remain cool enough for $\mathrm{C}_{2}$ and/or $\mathrm{CN}$ molecules to form (Green 2013). Most of the $\mathrm{CH}, \mathrm{Ba}$, and CEMP stars are brighter than the turnoff $\left(M_{G}<5\right)$ and may well be evolved dC stars. We have not corrected for reddening on this plot; interstellar reddening is insignificant for most dCs. However, the reddest giants $\left(B_{p}-R_{p} \gtrsim 1.8\right)$ are at low galactic latitude and likely significantly reddened. A cross-correlation with the Chandra CSC2.0 (Evans et al. 2010) and XMM-Newton 3XMM-DR8 (Rosen et al. 2016) catalogs reveals no new matches among these stars. 
Table 1

Chandra X-Ray Observations

\begin{tabular}{|c|c|c|c|c|c|c|}
\hline Object & Type & ObsID & Obs-Date & $\begin{array}{l}\text { Exposure } \\
\text { (ks) }\end{array}$ & Inst. & Mode \\
\hline SDSS J090128.28+323833.5 & $\mathrm{dCe}$ & 16650 & 2015 Dec 12 & 18.3 & ACIS-S & $\mathrm{VF}$ \\
\hline SDSS J101548.90+094649.7 & $\mathrm{DA} / \mathrm{dC}$ & 15702 & 2015 Jan 18 & 15.9 & ACIS-S & $\mathrm{F}$ \\
\hline \multirow{2}{*}{ SDSS J125017.9+252427.6 } & & 2976 & 2002 Dec 2 & 24.6 & ACIS-S & $\mathrm{F}$ \\
\hline & & 17461 & 2016 May 9 & 29.5 & ACIS-I & VF \\
\hline SDSS J151905.90+500702.9 & $\mathrm{DA} / \mathrm{dC}$ & 16649 & 2016 May 16 & 27.6 & ACIS-S & $\mathrm{VF}$ \\
\hline SDSS J163718.64+274026.5 & & 16652 & 2014 Nov 21 & 17.2 & ACIS-S & VF \\
\hline
\end{tabular}

Our X-ray analysis is very unlikely to be complicated by emission from the WDs, even in the $\mathrm{DA}+\mathrm{dC}$ systems, because hot WD photospheric X-ray emission is extremely soft. For WDs in the field, emission of hard X-rays is almost always attributed to coronal emission from a late-type dwarf companion (O'Dwyer et al. 2003). Indeed, among the thousands of WDs that have been observed (mostly serendipitously) by XMM-Newton or ROSAT, only a handful of single WDs have spectra that are hard like the CSPN (Bilíková et al. 2010). The great majority of the CSPNe with hard X-ray emission that do not have an easily detected late-type companion may have, e.g., an accreting WD companion (Miszalski et al. 2019).

Hydrogen Balmer line emission from the stellar chromosphere is a known tracer of activity; $\mathrm{H} \alpha$ line luminosity is well correlated with projected rotation velocity $v \sin i$ (e.g., Maldonado et al. 2017 for M0-M4 dwarfs) after excluding spectroscopic binaries. While all of the $\mathrm{dCs}$ in our sample have $\mathrm{H} \alpha$ emission evident by selection, we are unable to reliably use the $\mathrm{H} \alpha$ equivalent widths in these $\mathrm{dCs}$ to predict their X-ray emission. $\mathrm{H} \alpha$ equivalent widths are not a suitable indicator of stellar activity and must be transformed into $\mathrm{H} \alpha$ fluxes, as found by multiple studies (Reiners et al. 2012; Maldonado et al. 2017), before fitting $\mathrm{H} \alpha$-X-ray relations. However the correlation between $\mathrm{H} \alpha$ flux and X-ray flux appears to be a weak one even in $\mathrm{K}$ and $\mathrm{M}$ dwarfs and becomes only more problematic with $\mathrm{dCs}$ given the lack of published model atmospheres needed to transform $\mathrm{H} \alpha$ equivalent width into $\mathrm{H} \alpha$ flux. We therefore did not attempt to use the observed $\mathrm{H} \alpha$ emission to predict X-ray fluxes.

\section{Bolometric Luminosities}

Bolometric luminosity estimates are important for placing $\mathrm{dCs}$ in the context of other active stars, because stellar activity is best characterized in the X-rays across a range of stellar types when the X-ray luminosity is normalized by the bolometric luminosity and $L_{\text {bol }}$ can vary by a factor of $\sim 20$ across the range of main-sequence spectral types known to show $\mathrm{C}_{2}$ and $\mathrm{CN}$ molecular bands. To estimate bolometric luminosities $L_{\mathrm{bol}}$, we assembled a spectral energy distribution (SED) for each $\mathrm{dC}$ star in our sample from public cataloged photometry and calculated $L_{\text {bol }}$ using the sedkit Python package (Filippazzo et al. 2015; J. C. Filippazzo et al. 2019, in preparation). The exact procedure is detailed in those works and summarized here.
Table 2

X-Ray Source Properties in the 0.3-3.0 keV Energy Range

\begin{tabular}{|c|c|c|c|c|c|c|}
\hline Object & $\begin{array}{c}\text { Chandra } \\
\text { ObsID }\end{array}$ & $\begin{array}{c}\text { Net CR } \\
\left(\mathrm{cnt} \mathrm{ks}^{-1}\right)\end{array}$ & $\begin{array}{c}T_{\mathrm{X}} \\
(\mathrm{MK})\end{array}$ & $\begin{array}{l}F_{\mathrm{X}, \mathrm{obs}} \\
\left(10^{-15} \mathrm{erg}\right.\end{array}$ & $\left.\mathrm{cm}^{-2} \mathrm{~s}_{\mathrm{X}}{ }^{-1}\right)$ & $\begin{array}{c}L_{\mathrm{X}} \\
\left(10^{28} \mathrm{erg} \mathrm{s}^{-1}\right)\end{array}$ \\
\hline J0901 & 16650 & $0.16 \pm 0.09$ & 2 & $3.18_{-2.25}^{+4.33}$ & $3.77_{-2.67}^{+5.14}$ & $15.47_{-11.02}^{+21.12}$ \\
\hline$\ldots$ & $\ldots$ & $\ldots$ & 10 & $0.97_{-0.69}^{+1.33}$ & $1.03_{-0.73}^{+1.41}$ & $4.23_{-3.01}^{+5.79}$ \\
\hline $\mathrm{J} 1015$ & 15702 & $0.25 \pm 0.13$ & 2 & $3.64_{-2.30}^{+4.00}$ & $4.82_{-3.04}^{+5.28}$ & $13.02_{-8.25}^{+14.28}$ \\
\hline$\ldots$ & $\ldots$ & $\ldots$ & 10 & $1.34_{-0.85}^{+1.48}$ & $1.48_{-0.93}^{+1.63}$ & $4.00_{-2.53}^{+4.41}$ \\
\hline $\mathrm{J} 1250$ & 409 & $1.62 \pm 0.99$ & 2 & $20.40_{-15.08}^{+29.00}$ & $22.90_{-16.93}^{+32.50}$ & $21.91_{-16.20}^{+31.09}$ \\
\hline$\ldots$ & $\ldots$ & $\ldots$ & 10 & $8.48_{-6.27}^{+12.02}$ & $8.83_{-6.53}^{+12.57}$ & $8.45_{-6.25}^{+12.03}$ \\
\hline$\ldots$ & 2976 & $0.64 \pm 0.17$ & 2 & $12.80_{-5.00}^{+6.50}$ & $14.40_{-5.64}^{+7.30}$ & $13.78_{-5.40}^{+6.99}$ \\
\hline$\ldots$ & $\ldots$ & $\ldots$ & 10 & $4.13_{-1.61}^{+2.11}$ & $4.31_{-1.68}^{+2.19}$ & $4.12_{-1.61}^{+2.10}$ \\
\hline$\ldots$ & 17461 & $0.41 \pm 0.12$ & 2 & $47.30_{-19.70}^{+27.00}$ & $53.10_{-22.10}^{+30.30}$ & $50.80_{-21.17}^{+29.01}$ \\
\hline$\cdots$ & $\ldots$ & $\ldots$ & 10 & $6.03_{-2.51}^{+3.45}$ & $6.28_{-2.62}^{+3.59}$ & $6.01_{-2.51}^{+3.44}$ \\
\hline J1519 & 16649 & $0.54 \pm 0.14$ & 2 & $12.80_{-4.77}^{+6.30}$ & $14.40_{-5.39}^{+7.10}$ & $33.83_{-12.72}^{+16.72}$ \\
\hline .. & $\ldots$ & $\ldots$ & 10 & $3.52_{-1.31}^{+1.75}$ & $3.67_{-1.37}^{+1.81}$ & $8.62_{-3.23}^{+4.26}$ \\
\hline J1548 & 16651 & $0.31 \pm 0.14$ & 2 & $5.35_{-3.14}^{+5.15}$ & $6.00_{-3.52}^{+5.80}$ & $3.84_{-2.25}^{+3.71}$ \\
\hline ... & $\ldots$ & $\ldots$ & 10 & $0.26_{-0.26}^{+1.06}$ & $0.27_{-0.27}^{+1.10}$ & $0.17_{-0.17}^{+0.70}$ \\
\hline $\mathrm{J} 1637$ & 16652 & $0.57 \pm 0.18$ & 2 & $7.90_{-3.49}^{+5.00}$ & $11.00_{-4.85}^{+7.00}$ & $21.65_{-9.58}^{+13.80}$ \\
\hline .. & $\ldots$ & $\ldots$ & 10 & $3.03_{-1.34}^{+1.91}$ & $3.40_{-1.50}^{+2.15}$ & $6.69_{-2.96}^{+4.24}$ \\
\hline$\ldots$ & 17534 & $1.05 \pm 0.35$ & 2 & $14.20_{-6.59}^{+9.50}$ & $19.80_{-9.20}^{+13.20}$ & $38.97_{-18.16}^{+26.02}$ \\
\hline$\ldots$ & $\ldots$ & $\ldots$ & 10 & $5.50_{-2.55}^{+3.68}$ & $6.18_{-2.86}^{+4.12}$ & $12.16_{-5.65}^{+8.12}$ \\
\hline
\end{tabular}

Optical, near-infrared, and mid-infrared magnitudes were obtained from the SDSS Data Release 12 (Alam et al. 2015), 2MASS Point Source Catalog (Skrutskie et al. 2006), and AllWISE Source Catalog (Cutri 2013), respectively. We converted photometry to the VegaMag system and corrected for (small) interstellar extinction using the dustmaps Python package (Green 2018). Synthetic magnitudes were calculated from the SDSS spectrum, which was then normalized to the observational photometry. In wavelength regions with no spectral coverage, the SED was linearly interpolated between photometric points. For the DA/dCs, we excluded the SDSS $u^{-}, g_{-}$, and $r$-band photometry and spectral flux, since they are contaminated by the DA WDs. To approximate a Wien tail, the SED was linearly interpolated from the shortest-wavelength data point down to zero flux at zero wavelength. A blackbody spectrum fit to the WISE photometry was used to approximate a Rayleigh-Jeans tail at long wavelengths. We then calculated the bolometric flux $f_{\text {bol }}$ as the integral of the complete SED.

Finally, we calculated $L_{\text {bol }}=4 \pi f_{\text {bol }} d^{2}$ for each source using a parallax measurement from the Gaia DR2 and the resulting 


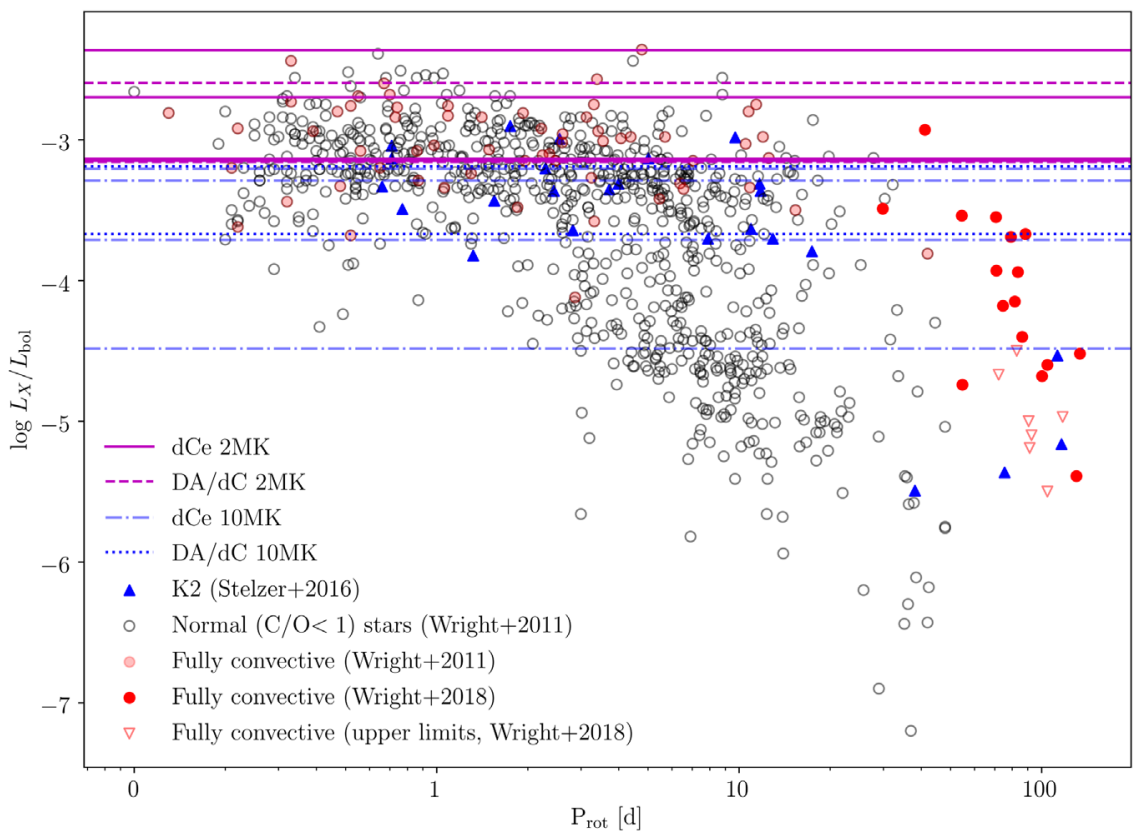

Figure 3. Activity level of $\mathrm{dC}$ stars in our sample in context with activity in normal $(\mathrm{C} / \mathrm{O}<1)$ main-sequence stars. The logarithm of $\mathrm{X}$-ray to bolometric luminosity ratio $\log L_{\mathrm{x}} / L_{\mathrm{bol}}$ is plotted vs. rotation period for the normal dwarf stars in the sample of Wright et al. (2011; open black circles). Fully convective dwarfs are highlighted from Wright et al. (2018; red circles, or red triangles for the upper limits) and from Wright et al. (2011; faint red circles). Stars from the Kepler Two-Wheel $(K 2)$ samples of Stelzer et al. (2016) are shown as blue triangles. The horizontal magenta (blue) lines show the $\log L_{\mathrm{x}} / L_{\mathrm{bol}}$ values for the six dC stars observed by Chandra in our pilot program, assuming a plasma temperature of $T_{\mathrm{X}}=2 \mathrm{MK}\left(T_{\mathrm{X}}=10 \mathrm{MK}\right.$ ). Dashed (dotted) lines represent the two DA/dC systems (with a hot white dwarf still visible in the optical spectrum). Solid (dotted-dashed) lines are for the other $\mathrm{dC}$ systems.

Table 3

Parallaxes, Distances, and Bolometric Luminosities

\begin{tabular}{lccc}
\hline \hline Object & $\begin{array}{c}\varpi \\
(\mathrm{mas})\end{array}$ & $\begin{array}{c}\text { Distance } \\
(\mathrm{pc})\end{array}$ & $\begin{array}{c}\log \left(L_{\mathrm{bol}} / L_{\odot}\right) \\
\left(\mathrm{erg} \mathrm{s}^{-1}\right)\end{array}$ \\
\hline $\mathrm{J} 0901$ & $1.71 \pm 0.14$ & $577.1 \pm 46.5$ & $-1.26 \pm 0.07$ \\
$\mathrm{~J} 1015$ & $2.10 \pm 0.13$ & $469.9 \pm 28.6$ & $-1.32 \pm 0.05$ \\
$\mathrm{~J} 1250$ & $3.54 \pm 0.08$ & $280.6 \pm 6.3$ & $-1.52 \pm 0.02$ \\
$\mathrm{~J} 1519$ & $2.26 \pm 0.08$ & $438.0 \pm 15.0$ & $-1.47 \pm 0.03$ \\
$\mathrm{~J} 1548$ & $4.32 \pm 0.08$ & $229.9 \pm 4.3$ & $-1.87 \pm 0.07$ \\
$\mathrm{~J} 1637$ & $2.47 \pm 0.09$ & $401.6 \pm 15.1$ & $-1.56 \pm 0.03$ \\
\hline
\end{tabular}

distances from Bailer-Jones et al. (2018). The results are shown in Table 3, where we adopt the solar bolometric luminosity value of $\log L_{\odot}=33.583$.

\section{Rotation-Activity Relationship}

Field main-sequence stars with outer convection zones are magnetically active, as traced by coronal X-rays and/or chromospheric $\mathrm{H} \alpha$ emission. The large observed range in $\log L_{\mathrm{x}} / L_{\text {bol }}$ from about $10^{-3}$ down to $10^{-8}$, is thought to occur because stars' rotation rates slow with age, due to mass loss from stellar winds (e.g., Skumanich 1972). Indeed, rotation rates correlate strongly with activity, especially when normalized by the convective turnover time $\tau$ via the Rossby number $R_{0}=P_{\text {rot }} / \tau$ (Noyes et al. 1984). The magnetic dynamo that drives these signatures of activity is thought to be generated by differential rotation inside the star. At the highest rotation rates, corresponding to $R_{0} \lesssim 0.13$, activity saturates, and $\log L_{\mathrm{x}} / L_{\mathrm{bol}}$ remains at $\sim-3.3$ (Micela et al. 1985; Wright et al. 2011). It was long thought that this correlation must be mediated by an $\alpha \Omega$ dynamo (Parker 1955), which requires an interface (the tacholine) between a solidly rotating radiative core and a differentially rotating convective envelope. However, the same rotation-activity relationship appears to hold even for late-type stars thought to be fully convective (Wright et al. 2018).

Unfortunately, we do not have the sensitive multiepoch photometry that would be required to measure the rotation rate for the $\mathrm{dC}$ stars in our sample. The greatest promise to achieve this in the near future-at least for the brighter $\mathrm{dC}$ examplesis after NASA's Transiting Exoplanet Survey Satellite (Ricker et al. 2015) surveys the northern sky, providing photometric measurements every 30 minutes, which can be combined as needed for greater sensitivity (at the cost of lower time resolution). Nor do we have estimates for the convective turnover times, due to a paucity of stellar structure models for $\mathrm{dC}$ stars. However, since we have empirical measurements of both $L_{\mathrm{x}}$ and $L_{\mathrm{bol}}$, we can estimate the range of likely values of $P_{\text {rot }}$ or $R_{0}$ for $\mathrm{dC}$ stars, reasonably assuming that their internal dynamics and magnetic dynamo have stabilized since the end of mass transfer.

In Figure 3, we show the activity level of $\mathrm{dC}$ stars in our sample as a function of rotation period, in context with activity in normal $(\mathrm{C} / \mathrm{O}<1)$ main-sequence stars from several recent sources in the literature. Wright et al. (2018) studied the coronal activity-rotation relationship for late-type stars with rotation periods known from the MEarth project (Nutzman \& Charbonneau 2008), including main-sequence stars thought to be fully convective- those later than about M3 $\left(T_{\mathrm{Eff}} \lesssim 3300 \mathrm{~K}\right)$, corresponding to masses below about $\sim 0.35 M_{\odot}$ (Chabrier \& Baraffe 1997). Stelzer et al. (2016) compiled X-ray data from ROSAT and XMM-Newton catalogs for K2 Superblink stars with well-measured periods. They assumed a thermal (APEC) single temperature model (APEC) of $3.5 \mathrm{MK}$ and a column density of $10^{19}$ atoms cm $\mathrm{cm}^{-2}$.

From Figure 3, limits on the rotation periods for these $\mathrm{dC}$ stars appear to be in the "saturated" regime where log 
$L_{\mathrm{x}} / L_{\mathrm{bol}} \geqslant-3.3$, at least when assuming plasma temperatures of $\sim 2 \mathrm{MK}$. About half remain in the saturated regime even when assuming a high plasma temperature $\sim 10 \mathrm{MK}$. If the lower temperature applies, then dCs' strong X-ray activity is consistent with rapid rotation rates of several days or less, as might be expected from accretion spin-up. If the higher temperature applies, then periods are more weakly constrained, to about 20 days or less.

We reiterate the caveats that (a) this pilot sample is not representative of $\mathrm{dCs}$ more generally, since, as mentioned, all six $\mathrm{dC}$ optical spectra in our sample show weak $\mathrm{H} \alpha$ emission lines (see Figure 1) and (b) X-ray counts are too few to actually constrain $T_{\mathrm{X}}$.

\section{Summary}

We have sought to test the hypothesis that $\mathrm{dC}$ stars, having inherited significant mass from an extinct AGB companion, could have spun up significantly, and that they may therefore show signs of coronal activity often associated with rapid rotation. As a test for such coronal activity, we selected a small sample of dCs for X-ray observation with Chandra. All six dC systems observed were detected by Chandra, but with too few counts to allow useful spectral constraints. We therefore calculate X-ray fluxes assuming plasma temperatures of 2 and $10 \mathrm{MK}$, bracketing a range of reasonable values. We have estimated the dCs' bolometric luminosities directly by compiling and integrating multiwavelength photometry and using distances from Gaia DR2 parallaxes published after our Chandra observations were performed. When assuming a reasonable plasma temperature of $2 \mathrm{MK}$, all six systems appear to have $\log L_{\mathrm{x}} / L_{\text {bol }} \gtrsim-3$, putting them in the saturated regime so that they might be expected to have short ( $\lesssim 10$ days) rotation periods consistent with strong accretion spin-up. At the upper range of expected temperature, near $10 \mathrm{MK}$, the X-ray activity of dCs could imply longer $(\lesssim 20$ days $)$ periods similar to less active dwarfs.

The X-ray emission from $\mathrm{dC}$ stars to date supports the masstransfer hypothesis. Where $\mathrm{dCs}$ have been part of a central binary system of a PN, which could be common, then the activity we detect here argues for spin-down significantly longer than $\left(\sim 10^{4} \mathrm{yr}\right) \mathrm{PN}$ timescales, because for most $\mathrm{dC}$ stars the former CSPNe are now WDs cooled beyond detectability.

To better understand the characteristics of a more typical sample, sufficiently sensitive X-ray observations of the closest known dC stars with reliable distances from Gaia DR2 are critical. Ideally, at least $\sim 100$ counts per object should be obtained, sufficient to constrain individual plasma temperatures to within about $\sim 30 \%$. The X-ray luminosity fractions $L_{\mathrm{x}} / L_{\mathrm{bol}}$ can then serve as a more reliable proxy to measure rotation and total accretion, within the context of models predicting longevity and intensity of the binary interaction during the AGB phase. For instance, accretion of both mass and angular momentum is enhanced if there is a longer RLOF phase before the CE phase. A dC enriched by a prolonged period of RLOF would have relatively stronger $\mathrm{X}$-ray emission, faster rotation, and perhaps also higher $\mathrm{C} / \mathrm{O}$. Very close $\mathrm{PCEB} \mathrm{dC}$ systems that have low $\mathrm{X}$-ray activity may indicate a brief pre-CE phase. A dC that experienced only wind accretion may have low rotation and low $L_{\mathrm{X}}$. A significant sample of $\mathrm{dCs}$ with measured orbital and rotation periods and X-ray luminosities would greatly enhance our understanding of these useful and intriguing systems.
The scientific results reported in this article are based on observations made by the Chandra X-ray Observatory, and data obtained from the Chandra Data Archive. This research has made use of software provided by the Chandra X-ray Center (CXC) in the application packages CIAO and Sherpa.

Support for this work was provided by the National Aeronautics and Space Administration through Chandra Award Numbers GO4-15005X and GO5-16004X issued by the Chandra X-ray Center, which is operated by the Smithsonian Astrophysical Observatory for and on behalf of the National Aeronautics Space Administration under contract NAS8-03060. B.M. acknowledges support from the National Research Foundation (NRF) of South Africa.

Funding for the Sloan Digital Sky Survey IV has been provided by the Alfred P. Sloan Foundation, the U.S. Department of Energy Office of Science, and the Participating Institutions. SDSS acknowledges support and resources from the Center for High-Performance Computing at the University of Utah. The SDSS website is www.sdss.org.

SDSS is managed by the Astrophysical Research Consortium for the Participating Institutions of the SDSS Collaboration including the Brazilian Participation Group, the Carnegie Institution for Science, Carnegie Mellon University, the Chilean Participation Group, the French Participation Group, Harvard-Smithsonian Center for Astrophysics, Instituto de Astrofísica de Canarias, The Johns Hopkins University, Kavli Institute for the Physics and Mathematics of the Universe (IPMU)/University of Tokyo, Lawrence Berkeley National Laboratory, Leibniz Institut für Astrophysik Potsdam (AIP), Max-Planck-Institut für Astronomie (MPIA Heidelberg), Max-Planck-Institut für Astrophysik (MPA Garching), MaxPlanck-Institut für Extraterrestrische Physik (MPE), National Astronomical Observatories of China, New Mexico State University, New York University, University of Notre Dame, Observatório Nacional/MCTI, The Ohio State University, Pennsylvania State University, Shanghai Astronomical Observatory, United Kingdom Participation Group, Universidad Nacional Autónoma de México, University of Arizona, University of Colorado Boulder, University of Oxford, University of Portsmouth, University of Utah, University of Virginia, University of Washington, University of Wisconsin, Vanderbilt University, and Yale University.

Facilities: 2MASS, CXO, Gaia, Sloan, WISE.

Software: Astropy (Astropy Collaboration et al. 2018), matplotlib (Barrett et al. 2005), Numpy (http://www. tramy.us/).

\section{Appendix X-Ray Spectral Models}

Our motivation for using 2 and $10 \mathrm{MK}$ plasma temperatures for the X-ray spectral models of $\mathrm{dC}$ stars comes both from the literature and from our Chandra observations. The highertemperature model is consistent with median photon energies of $\sim 0.5-1.0 \mathrm{keV}(6-12 \mathrm{MK})$ seen in X-ray-selected stellar samples observed with Chandra from, e.g., the COSMOS survey (Wright et al. 2010), and also with the temperature of peak emissivity for Mg XI. However, for some single stars with detailed X-ray spectral fitting, plasma temperatures are close to 2 MK (e.g., see the compilation in Table 9 of Testa et al. 2004), the temperature of peak emissivity for O VII.

Even with very few counts detected in our Chandra images, we can examine the median energy values and their location 


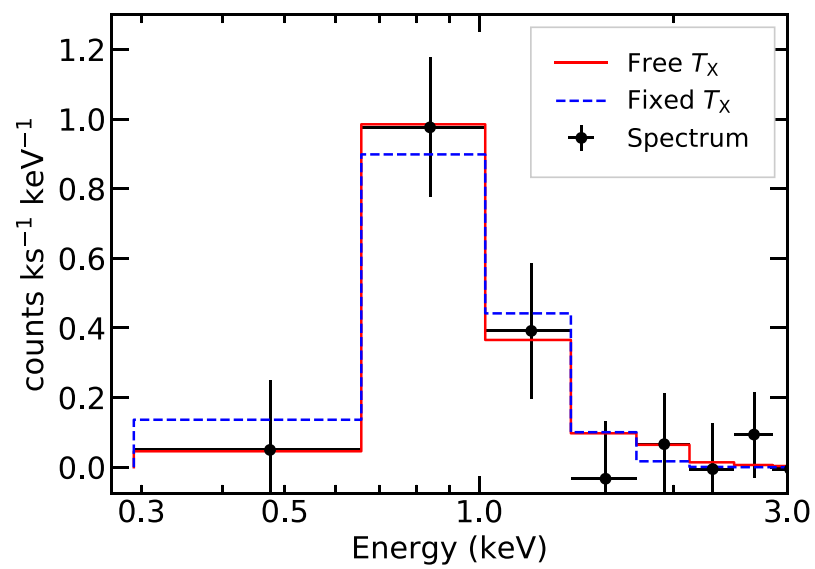

(a) Counts spectrum

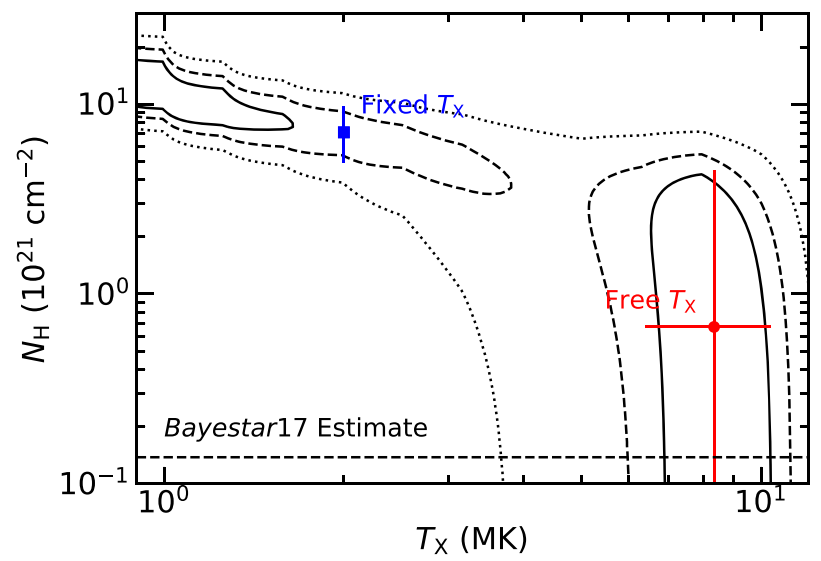

(b) Best-fit X-ray spectral parameters

Figure 4. Chandra spectrum of SDSS J151905.90+500702.9 from ObsID 16649. (a) The photon spectrum in counts ks ${ }^{-1} \mathrm{keV}^{-1}$. The expected counts for both $2 \mathrm{MK}$ and $8 \mathrm{MK}$ temperature plasma models are overplotted as blue dashed and red solid lines, respectively. Since the total number of net counts is only $\sim 15$, the fits are equally good and quite poorly constrained. (b) The best-fit contours (at $68.3 \%, 90 \%$, and $99 \%$ ) for the APEC spectral fit parameters of temperature $T_{\mathrm{X}}$ and intervening hydrogen column $N_{\mathrm{H}}$. The intervening column estimated from Bayestar17 (Green 2018) is quite low (dashed horizontal line) and is consistent with the best-fit spectrum for plasma temperature near $\sim 8 \mathrm{MK}$. If we fix the temperature at $T_{\mathrm{X}}=2 \mathrm{MK}$, a larger column is required, which may be consistent with possible circumstellar material.

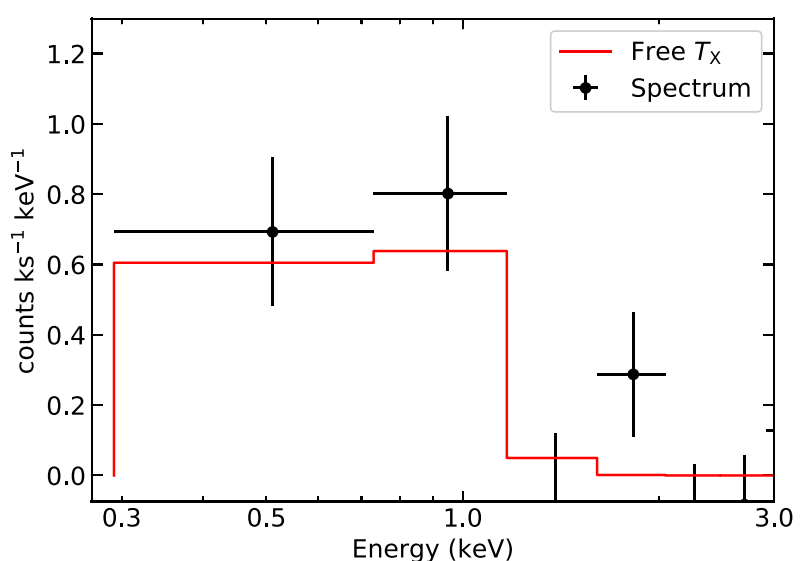

(a) Counts spectrum

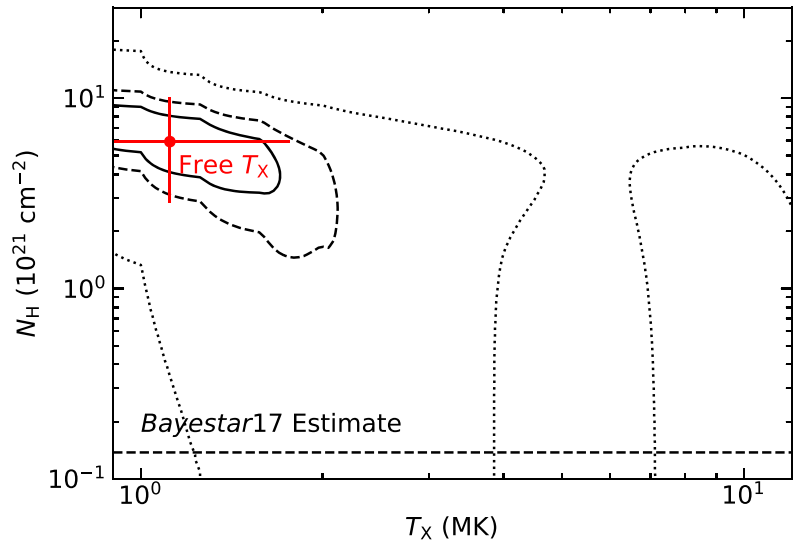

(b) Best-fit X-ray spectral parameters

Figure 5. Chandra spectrum of SDSS J125017.9+252427.6 from ObsID 2976. (a) The photon spectrum in counts ks ${ }^{-1} \mathrm{keV}^{-1}$. The expected counts for the best-fit $\sim 1$ MK APEC model is overplotted in red. (b) As in Figure 4, the APEC spectral fit parameter contours of temperature $T_{\mathrm{X}}$ and intervening hydrogen column $N_{\mathrm{H}}$. The best-fit temperature is near $\sim 1 \mathrm{MK}$, but requires an intervening column density much larger than expected from the ISM, broadly consistent with possible circumstellar material.

with respect to the thermal models and foreground extinction. Most of the observed counts suggest $T_{\mathrm{X}}>3 \mathrm{MK}$ and likely near $8-10 \mathrm{MK}$, but this is uncertain without knowing what the circumstellar environment might contribute to extinction.

We can also analyze the two dCs in our sample with the largest number of counts, which are nonetheless insufficient for strong spectral model constraints. SDSS J151905.90+500702.9 (Figure 4) indicates that either a hot plasma temperature ( $\sim 10 \mathrm{MK}$ with low absorbing column, $N_{\mathrm{H}}<5 \times 10^{21} \mathrm{~cm}^{-2}$ ) or a cool plasma temperature $(\lesssim 2 \mathrm{MK}$, with high absorbing column, $N_{\mathrm{H}}>5 \times 10^{21} \mathrm{~cm}^{-2}$ ) might be applicable. The best-fit spectral model for SDSS J125017.9+252427.6 (Figure 5) is single-peaked toward a cool value near $\sim 1 \mathrm{MK}$.

These crude spectral fits further justify our adopted spectral models of 2 and $10 \mathrm{MK}$. Formally they may allow for the possibility of significant circumstellar columns above the expected intervening values, but the constraints are very weak. Cool post-AGB wind material may indeed surround dCs, but such an intriguing possibility must be investigated with much more sensitive X-ray and/or submillimeter observations (e.g., Bujarrabal et al. 2013) before being taken seriously.

\section{ORCID iDs}

Paul J. Green (1) https://orcid.org/0000-0002-8179-9445

Rodolfo Montez (i) https://orcid.org/0000-0002-6752-2909

Joseph Filippazzo (1) https://orcid.org/0000-0002-0201-8306

Jeremy J. Drake (i) https://orcid.org/0000-0002-0210-2276

Jay Farihi (1) https://orcid.org/0000-0003-1748-602X

Joel H. Kastner (1) https://orcid.org/0000-0002-3138-8250

Benjamin R. Roulston (i) https://orcid.org/0000-00029453-7735

\section{References}

Abate, C., Pols, O. R., \& Stancliffe, R. J. 2018, A\&A, 620, A63 Afşar, M., \& Ibanoğlu, C. 2008, MNRAS, 391, 802 
Alam, S., Albareti, F. D., Allende Prieto, C., et al. 2015, ApJS, 219, 12 Astropy Collaboration, Price-Whelan, A. M., Sipőcz, B. M., et al. 2018, AJ, 156,123

Bailer-Jones, C. A. L., Rybizki, J., Fouesneau, M., et al. 2018, AJ, 156, 58

Balick, B. 2004, AJ, 127, 2262

Balick, B., \& Frank, A. 2002, ARA\&A, 40, 439

Barrett, P., Hunter, J., Miller, J. T., et al. 2005, in ASP Conf. Ser. 347 Astronomical Data Analysis Software and Systems XIV, ed. P. Shopbell, M. Britton, \& R. Ebert (San Francisco, CA: ASP), 91

Bilíková, J., Chu, Y.-H., Gruendl, R. A., et al. 2010, AJ, 140, 1433

Blackman, E. G., Frank, A., \& Welch, C. 2001, ApJ, 546, 288

Bromm, V., \& Loeb, A. 2003, Nature, 425, 812

Bujarrabal, V., Alcolea, J., Van Winckel, H., et al. 2013, A\&A, 557, A104

Chabrier, G., \& Baraffe, I. 1997, A\&A, 327, 1039

Chen, Z., Blackman, E. G., Nordhaus, J., Frank, A., \& Carroll-Nellenback, J. 2018, MNRAS, 473, 747

Corradi, R. L. M., Sabin, L., Miszalski, B., et al. 2011, MNRAS, 410, 1349 Cutri, R. M. 2013, yCat, 2328

Dahn, C. C., Liebert, J., Kron, R. G., Spinrad, H., \& Hintzen, P. M. 1977, ApJ, 216,757

de Kool, M., \& Green, P. J. 1995, ApJ, 449, 236

Dearborn, D. S. P., Liebert, J., Aaronson, M., et al. 1986, ApJ, 300, 314

Downes, R. A., Margon, B., Anderson, S. F., et al. 2004, AJ, 127, 2838

Evans, I. N., Primini, F. A., Glotfelty, K. J., et al. 2010, ApJS, 189, 37

Farihi, J., Arendt, A. R., Machado, H. S., \& Whitehouse, L. J. 2018, MNRAS, 477, 3801

Filippazzo, J. C., Rice, E. L., Faherty, J., et al. 2015, ApJ, 810, 158

Fortney, J. J. 2012, ApJL, 747, L27

Frew, D. J. 2008, PhD thesis, Macquarie Univ.

Gaia Collaboration, Brown, A. G. A., Vallenari, A., et al. 2018, A\&A, 616, A1

García-Segura, G., Ricker, P. M., \& Taam, R. E. 2018, ApJ, 860, 19

Garraffo, C., Drake, J. J., Alvarado-Gomez, J. D., et al. 2018, ApJ, 868, 60

Gondoin, P. 2018, A\&A, 616, A154

Green, G. M. 2018, JOSS, 3, 695

Green, P. 2013, ApJ, 765, 12

Green, P. J., Margon, B., \& MacConnell, D. J. 1991, ApJL, 380, L31

Heber, U., Bade, N., Jordan, S., \& Voges, W. 1993, A\&A, 267, L31

Iaconi, R., De Marco, O., Passy, J.-C., et al. 2018, MNRAS, 477, 2349

Jeffries, R. D., \& Stevens, I. R. 1996, MNRAS, 279, 180

Ji, W., Cui, W., Liu, C., et al. 2016, ApJS, 226, 1

Jones, D., Boffin, H. M. J., Rodríguez-Gil, P., et al. 2015, A\&A, 580, A19

Jones, D., Van Winckel, H., Aller, A., Exter, K., \& De Marco, O. 2017, A\&A, 600, L9

Kastner, J. H., Montez, R., Balick, B., et al. 2012, AJ, 144, 58

Kosovichev, A. G., de Gouveia Dal Pino, E., \& Yan, Y. 2013, Solar and Astrophysical Dynamos and Magnetic Activity (Cambridge: Cambridge Univ. Press)

Kraft, R. P. 1967, ApJ, 150, 551

Li, Y.-B., Luo, A.-L., Du, C.-D., et al. 2018, ApJS, 234, 31

Liebert, J., Schmidt, G. D., Lesser, M., et al. 1994, ApJ, 421, 733

Lucatello, S., Tsangarides, S., Beers, T. C., et al. 2005, ApJ, 625, 825

Maldonado, J., Scandariato, G., Stelzer, B., et al. 2017, A\&A, 598, A27

Margon, B., Kupfer, T., Burdge, K., et al. 2018, ApJL, 856, L2
Matt, S. P., Brun, A. S., Baraffe, I., et al. 2015, ApJL, 799, L23

Micela, G., Sciortino, S., Serio, S., et al. 1985, ApJ, 292, 172

Miszalski, B., Acker, A., Moffat, A. F. J., et al. 2009, A\&A, 496, 813

Miszalski, B., Boffin, H. M. J., \& Corradi, R. L. M. 2013, MNRAS, 428, L39

Miszalski, B., Boffin, H. M. J., Frew, D. J., et al. 2012, MNRAS, 419, 39

Miszalski, B., Boffin, H. M. J., Jones, D., et al. 2013, MNRAS, 436, 3068

Miszalski, B., Manick, R., Mikołajewska, J., et al. 2018, MNRAS, 473, 2275

Miszalski, B., Manick, R., Van Winckel, H., et al. 2019, PASA, 36, e018

Montez, R., De Marco, O., Kastner, J. H., et al. 2010, ApJ, 721, 1820

Montez, R., Jr., Kastner, J. H., Balick, B., et al. 2015, ApJ, 800, 8

Morales, J. C., Ribas, I., \& Jordi, C. 2008, A\&A, 478, 507

Morgan, D. P., West, A. A., Garcés, A., et al. 2012, AJ, 144, 93

Morrison, R., \& McCammon, D. 1983, ApJ, 270, 119

Munn, J. A., Monet, D. G., Levine, S. E., et al. 2004, AJ, 127, 3034

Norris, J. E., Yong, D., Bessell, M. S., et al. 2013, ApJ, 762, 28

Noyes, R. W., Hartmann, L. W., Baliunas, S. L., Duncan, D. K., \& Vaughan, A. H. 1984, ApJ, 279, 763

Nutzman, P., \& Charbonneau, D. 2008, PASP, 120, 317

O’Dwyer, I. J., Chu, Y.-H., Gruendl, R. A., et al. 2003, AJ, 125, 2239

Oomen, G.-M., Van Winckel, H., Pols, O., et al. 2018, A\&A, 620, A85

Pallavicini, R., Golub, L., Rosner, R., et al. 1981, ApJ, 248, 279

Parker, E. N. 1955, ApJ, 122, 293

Plez, B., \& Cohen, J. G. 2005, A\&A, 434, 1117

Reichardt, T. A., De Marco, O., Iaconi, R., et al. 2019, MNRAS, 484, 631

Reiners, A., Joshi, N., \& Goldman, B. 2012, AJ, 143, 93

Ricker, G. R., Winn, J. N., Vanderspek, R., et al. 2015, JATIS, 1, 14003

Rosen, S. R., Webb, N. A., Watson, M. G., et al. 2016, A\&A, 590, A1

Roulston, B. R., Green, P. J., Ruan, J. J., et al. 2019, ApJ, 877, 44

Si, J., Luo, A., Li, Y., et al. 2014, SCPMA, 57, 176

Skrutskie, M. F., Cutri, R. M., Stiening, R., et al. 2006, AJ, 131, 1163

Skumanich, A. 1972, ApJ, 171, 565

Smith, M. C., Ruchti, G. R., Helmi, A., et al. 2007, MNRAS, 379, 755

Smith, R. K., Brickhouse, N. S., Liedahl, D. A., \& Raymond, J. C. 2001, ApJL, 556, L91

Soker, N., \& Kastner, J. H. 2002, ApJ, 570, 245

Starkenburg, E., Shetrone, M. D., McConnachie, A. W., et al. 2014, MNRAS, 441, 1217

Stelzer, B., Damasso, M., Scholz, A., \& Matt, S. P. 2016, MNRAS, 463, 1844

Stelzer, B., Marino, A., Micela, G., et al. 2013, MNRAS, 431, 2063

Testa, P., Drake, J. J., \& Peres, G. 2004, ApJ, 617, 508

Tielens, A. G. G. M. 2005, in ASP Conf. Ser. 341, Chondrites and the Protoplanetary Disk, ed. A. N. Krot, E. R. D. Scott, \& B. Reipurth (San Francisco, CA: ASP), 605

Tyndall, A. A., Jones, D., Boffin, H. M. J., et al. 2014, RMxAC, 44, 60

West, A. A., Hawley, S. L., Bochanski, J. J., et al. 2008, AJ, 135, 785

Whitehouse, L. J., Farihi, J., Green, P. J., Wilson, T. G., \& Subasavage, J. P. 2018, MNRAS, 479, 3873

Wood, P. R., Olivier, E. A., \& Kawaler, S. D. 2004, ApJ, 604, 800

Wright, N. J., Drake, J. J., \& Civano, F. 2010, ApJ, 725, 480

Wright, N. J., Drake, J. J., Mamajek, E. E., \& Henry, G. W. 2011, ApJ, 743, 48

Wright, N. J., Newton, E. R., Williams, P. K. G., Drake, J. J., \& Yadav, R. K. 2018, MNRAS, 479, 2351

Yoon, J., Beers, T. C., Placco, V. M., et al. 2016, ApJ, 833, 20 